\title{
Correlation of Contractile Dysfunction with Oxidative Energy Production and Tissue High Energy Phosphate Stores during Partial Coronary Flow Disruption in Rabbit Heart
}

\author{
Robert C. Marshall \\ With the technical assistance of Dan-Ya Zhang \\ Divisions of Cardiology, Departments of Medicine, University of California, Los Angeles (UCLA) School of Medicine, Los Angeles, \\ California 90024; Martinez Veterans Administration Medical Center, Martinez, California 94553; and University of California, Davis \\ School of Medicine, Davis, California 95616
}

\begin{abstract}
The relationships between contractile function, myocardial oxygen consumption, and tissue high energy phosphate and lactate content were investigated during partial coronary flow disruption. The experimental preparation was an isolated, isovolumic retrograde blood-perfused rabbit heart. Both developed pressure $(r=0.94)$ and $\mathrm{dp} / \mathrm{dt}(r=0.95)$ exhibited strong linear correlations with myocardial oxygen consumption that were stable for up to $\mathbf{4 5} \mathrm{min}$ after blood flow reduction. In contrast, tissue high energy phosphate content exhibited nonlinear relationships with both developed pressure and oxygen consumption such that systolic mechanical function and oxidative metabolism declined to 20 and $30 \%$ of control values, respectively, before significant abnormalities in myocardial high energy phosphate stores were observed. Similarly, developed pressure and oxygen consumption decreased to 36 and $48 \%$ of control, respectively, before abnormal tissue lactate content was detected. The results of this study indicate that: $(a)$ mechanical function is closely related to the rate of oxidative energy production during partial coronary flow disruption, and (b) despite the development of significant contractile dysfunction, tissue high energy phosphate content remains at normal levels except under the most severely flow-deprived conditions. The preservation of tissue energy stores can be explained by the apparent coupling of contractile performance to oxidative energy production, which could function to maintain myocardial energy balance during partial coronary flow restriction.
\end{abstract}

\section{Introduction}

Previous studies have demonstrated that the severity of myocardial contractile dysfunction correlates with the degree of blood flow impairment during myocardial ischemia produced by partial coronary artery obstruction (1-4). Based on the known effects of impaired oxygen delivery, it has been postulated that the close correlation between function and perfusion reflects corresponding abnormalities in tissue ATP content and/or oxidative ATP production $(1,5,6)$. However, the relationship between contractile performance and tissue energy

Address correspondence to Dr. Marshall, Veterans Administration Medical Center, 150 Muir Road, Martinez, CA 94553.

Received for publication 1 September 1987 and in revised form 8 February 1988.

J. Clin. Invest.

(C) The American Society for Clinical Investigation, Inc.

0021-9738/88/07/0086/10 \$2.00

Volume 82, July 1988, 86-95 metabolism has been evaluated only during oxygen deprivation produced by complete coronary artery occlusion or hypoxic/anoxic myocardial perfusion (7-13). Because of potential differences in the rate of oxygen delivery, residual oxidative energy production, and cellular washout, extrapolation of these results to incomplete coronary flow restriction seems unwarranted. In addition, prior investigations have not correlated oxygen-limited contractile performance with directly measured myocardial oxygen consumption (OC). ${ }^{1}$ Therefore, the potential correlation between contractile dysfunction, tissue high energy phosphate content, and oxidative energy production during partial coronary flow disruption has yet to be evaluated.

The purpose of the present study was to determine the relationships between contractile dysfunction, myocardial oxidative ATP production as assessed by oxygen consumption, and tissue high energy phosphate and lactate content during partial coronary flow restriction. To accomplish this goal, graded, steady state reductions in myocardial perfusion were produced in isolated, isovolumic, retrograde blood-perfused rabbit hearts. In this experimental preparation, coronary flow reductions are global and independent of ventricular loading conditions. Isovolumic developed pressure (DP) and its first derivative, myocardial $O C$ and tissue ATP, creatine phosphate (CrP), ADP, AMP, and lactate content were measured in hearts with flow rates ranging from a control value of $( \pm S E)$ $2.15 \pm 0.16 \mathrm{ml} / \mathrm{g}$ wet $\mathrm{wt} \mathrm{min}^{-1}$ to $0.17 \pm 0.01 \mathrm{ml} / \mathrm{g}$ wet $\mathrm{wt} \mathrm{min}^{-1}$. The results of this study indicate that flow-limited contractile performance and myocardial OC are linearly related while contractile performance and OC must decline to 20 and $30 \%$ of control values, respectively, before significant abnormalities in myocardial high energy phosphate content are detected. These observations suggest that the apparent coupling of mechanical performance and oxidative energy production might function to preserve myocardial energy balance during partial coronary flow restriction.

\section{Methods}

Experimental preparation. Preparation of isovolumically beating, retrograde blood-perfused rabbit hearts was similar to that previously reported (14-16). Briefly, after administration of $10 \mathrm{mg}$ of sodium heparin and $200 \mathrm{mg}$ of sodium pentobarbital via an ear vein, hearts from nonfasted male New Zealand white rabbits $(2.0-2.5 \mathrm{~kg})$ were excised through a median sternotomy and arrested in ice-cold saline. The aorta was rapidly cannulated to allow retrograde perfusion with red blood cell-containing perfusate. Flow was held constant at $\sim 2.0$

1. Abbreviations used in this paper: $\mathrm{CrP}$, creatine phosphate; $\mathrm{DP}$, developed pressure; $\mathrm{OC}$, oxygen consumption; TAN, total adenine nucleotide. 
$\mathrm{ml} / \mathrm{g}$ wet wt $\mathrm{min}^{-1}$ using a Minipuls 2 peristaltic pump (Gilson Co., Inc., Worthington, $\mathrm{OH}$ ). The red blood cell containing perfusate was not recirculated.

An apical drain was inserted through the left atrium into the left ventricle to allow drainage of fluid from the Thebesian circulation. After the atrioventricular node was crushed to allow controlled stimulation, a fluid-filled latex balloon connected to a Gould-Statham P23ID pressure transducer (Gould Inc., Oxnard, CA) was inserted into the left ventricle via the left atrium and mitral valve. A coronary venous sampling catheter and needle thermistor (Bailey Instrument Co., Inc., Saddlebrook, NJ) were inserted into the right ventricle via the right atrium. The venae cavae and pulmonary artery were then ligated so that all coronary venous drainage flowed out the sampling catheter without exposure to the atmosphere.

Hearts were perfused with a modified Tyrode's solution containing $20 \%$ oxygenated bovine red cells and $15 \mathrm{~g} /$ liter bovine serum albumin (free fatty acid free, Sigma Chemical Co., St. Louis, MO). The specific electrolyte concentrations were (in millimoles/liter or $\mathrm{mM}$ ): $\mathrm{NaCl}$, $110.0 ; \mathrm{CaCl}_{2}, 2.5 ; \mathrm{KCl}, 6.0 ; \mathrm{MgCl}_{2}, 1.0 ; \mathrm{NaH}_{2} \mathrm{PO}_{4}, 0.435$; and $\mathrm{NaHCO}_{3}, 28.0$. Bovine serum albumin was prepared by overnight dialysis at $4^{\circ} \mathrm{C}$ against a large volume of buffer and filtration through a $0.8-\mu \mathrm{m}$ Millipore filter. Dextrose $(11 \mathrm{mM})$, insulin $(2.5 \mathrm{mU} / \mathrm{ml})$, and sodium acetate $(2.0 \mathrm{mM})$ were provided to ensure adequate substrate availability to the myocardium. In one series of experiments, perfusate hematocrit was systematically reduced from 20 to $10 \%, 5 \%$, and $0 \%$.

Bovine erythrocytes were collected from a local slaughter house with sufficient heparin for anticoagulation, transported on ice, and immediately centrifuged $(2,600 \mathrm{~g})$ at $4^{\circ} \mathrm{C}$ for $20 \mathrm{~min}$. After aspirating plasma and buffy coat, the cells were washed three times and stored in calcium-free buffer. Red cells were oxygenated by washing five times in ice-cold perfusate which had been equilibrated with $100 \%$ oxygen. Final perfusate $\mathrm{pH}$ was adjusted to between 7.30 and 7.40 by equilibrating the buffer in which red cells were suspended for myocardial perfusion with $98 \% / 2 \% \mathrm{O}_{2} / \mathrm{CO}_{2}$. The red cell, albumin-containing perfusate was placed on ice and a mixture of $100 \% \mathrm{O}_{2}$ and $98 \% / 2 \%$ $\mathrm{O}_{2} / \mathrm{CO}_{2}$ was blown over the surface of the red cells in sufficient quantity to maintain oxygen content and $\mathrm{pH}$ constant. An in-line Swank transfusion filter ( $13 \mu \mathrm{m}$ exclusion; model IL 200; Pioneer Viggo, Inc., Beaverton, OR) was used to filter red cell aggregates.

Data collection and analytical procedures. Diastolic pressure, isovolumic DP, and the first derivative of DP were recorded continuously from the fluid-filled ventricular balloon on an HP7404A recorder (Hewlett Packard Co., Palo Alto, CA). The dynamic response characteristics of this pressure measurement system were characterized by suddenly releasing pressure from a hand-held syringe. The damped natural frequency and damping coefficient were $43 \mathrm{~Hz}$ and 0.59 , respectively. The calculated natural frequency was $54 \mathrm{~Hz}$, suggesting a "flat" response to $>40 \mathrm{~Hz}$. These results satisfy the criteria of Falsetti et al. (17) for accurate measurement of ventricular pressure and its first derivative using fluid-filled catheters.

Arterial and venous oxygen concentrations were determined on a Lex $\mathrm{O}_{2}$ Con oxygen analyzer (Lexington Instruments, Waltham, MA). Oxygen extraction was computed from the directly measured arterial and venous concentrations while the rate of oxygen consumption was determined using these concentrations and the predetermined flow rate. Arterial samples were taken from a side-arm placed just above the aortic cannula.

Analysis of tissue high energy phosphate and lactate content was performed by smash-freezing the left ventricular free wall with Wollenberger clamps cooled to the temperature of liquid nitrogen. Frozen myocardial wafers (width $0.8-1.5 \mathrm{~mm}$ ) were subsequently pulverized, extracted with $6 \%$ perchloric acid, and neutralized with $5 \mathrm{M} \mathrm{K}_{2} \mathrm{CO}_{3}$. Myocardial ATP and CrP were measured spectrophotometrically using the glucose-6-phosphate dehydrogenase enzyme assay, ADP and AMP, with the coupled pyruvate kinase-lactate dehydrogenase reaction and lactate with the lactate dehydrogenase procedure $(16,18)$.

Experimental protocol. After the preparation was complete, the left ventricular balloon was inflated until end-diastolic pressure was 8
mmHg. Stimulating electrodes from a Grass SD44 stimulator were placed against the left and right ventricles and 4-V, 4-ms stimuli were delivered at a rate of $180 / \mathrm{min}$. Both the balloon volume and stimulus rate were subsequently maintained constant so that ventricular loading conditions and heart rate were invariant throughout each experiment. Similarly, temperature was maintained between $37^{\circ}$ and $38^{\circ} \mathrm{C}$ using a water-jacketed perfusate heating coil and heart chamber.

An equilibration period of $15 \mathrm{~min}$ preceded any experimental intervention. At end equilibration, myocardial blood flow and ventricular performance were assessed to allow evaluation of the comparability of hearts in the different experimental subgroups. The results of this analysis revealed that there were no statistically significant preintervention differences in the rate of myocardial perfusion $(2.25 \pm 0.11$ $\mathrm{ml} / \mathrm{g}$ wet $\left.\mathrm{wt} \mathrm{min}^{-1}\right), \mathrm{DP}(102.7 \pm 1.4 \mathrm{mmHg})$, or end-diastolic pressure $(7.9 \pm 0.10 \mathrm{mmHg})$ between any of the experimental subgroups $(P$ $>0.05$ for all comparisons).

After equilibration, myocardial perfusion was reduced and subsequently held constant so that each heart was studied at only one myocardial perfusion rate. The particular flow rate evaluated in individual experiments was predetermined from an alternating schedule of stepwise reductions/increases in perfusion between control-flow and the lowest flow rate evaluated. Perfusion pressure averaged 57.1 \pm 2.5 $\mathrm{mmHg}$ under control conditions and declined in parallel with the severity of flow restriction, with the lowest pressure $(19 \pm 0.5 \mathrm{mmHg})$ recorded at the lowest flow rate. Balloon volume and stimulus rate were held constant in all hearts so that changes in the rate $\times$ pressure product and mechanical energy consumption would be accurately reflected by detected alterations in isovolumic developed pressure.

To assess the effect of time on the relationships between flow-restricted mechanical performance, $O C$, and tissue high energy phosphate and lactate content, two groups of hearts were evaluated. In the first group (group I, 30 hearts), varying severities of flow restriction were produced and held constant for $15 \mathrm{~min}$ before smash-freezing. In the second group (group II, 29 hearts), flow was reduced in an identical fashion as for group I and maintained constant for $45 \mathrm{~min}$ before smash-freezing. In both groups, the presence of steady state mechanical performance and oxygen utilization was evaluated by measuring OC, end-diastolic pressure, and DP and its first derivative at 5, 10, and 15 min after initial flow restriction. In group II hearts, an additional set of measurements was taken at $45 \mathrm{~min}$.

Data are expressed as mean \pm SEM. Statistical analysis of data acquired sequentially in individual hearts was performed using an analysis of variance with repeated measures while comparisons between experimental groups was performed with a two-way analysis of variance. The relationships between DP, its first derivative, $O C$, and myocardial high energy phosphate and lactate content were evaluated by obtaining best-fit curves using least-squares analysis of linear, polynomial, and exponential expressions. Significant differences between best-fit curves were determined by regression analysis. A $P$ value $<0.05$ was considered significant.

\section{Results}

Fig. 1 illustrates the relationship between flow, OC, and oxygen extraction during progressive flow restriction. The data have been organized into six subgroups according to the measured rates of flow-limited oxygen consumption as outlined in Table II. Under control conditions, flow, OC, and oxygen extraction fraction averaged $2.15 \pm 0.16 \mathrm{ml} / \mathrm{g}$ wet $\mathrm{wt} \mathrm{min}^{-1}$, $4.25 \pm 0.21 \mu \mathrm{mol} / \mathrm{g}$ wet $\mathrm{wt} \mathrm{min}^{-1}$, and $0.59 \pm 0.05$, respectively. As flow was reduced to a minimum value of $0.17 \pm 0.01 \mathrm{ml} / \mathrm{g}$ wet $\mathrm{wt} \mathrm{min}^{-1}$, oxygen extraction fraction increased to a maximum of $0.96 \pm 0.02$, while OC declined to $0.54 \pm 0.03 \mu \mathrm{mol} / \mathrm{g}$ wet $\mathrm{wt} \mathrm{min}^{-1}$. The virtually complete utilization of oxygen at low flows indicates that the decline in OC during progressive 


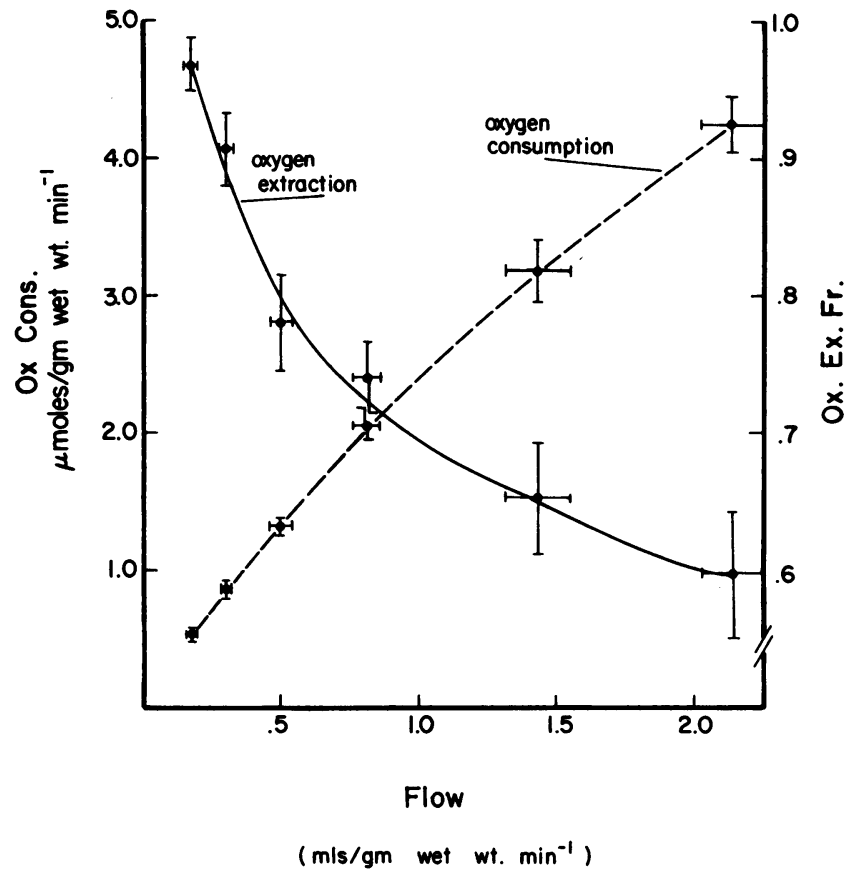

Figure 1. Relationship between myocardial OC (Ox Cons.), oxygen extraction, and blood flow during graded, steady-state reductions in myocardial perfusion. Data are the average results from the first 15 min for both group I and II hearts. Each data point is the mean \pm SEM for 9 to 12 hearts. Ox. Ex. Fr., oxygen extraction fraction. flow restriction was due to decreased oxygen delivery and not to a decline in the percent nutritional blood flow.

Fig. 2 illustrates the effect of time on the values for myocardial OC $(A), \mathrm{DP}(B), \mathrm{dp} / \mathrm{dt}(C)$, and diastolic pressure $(D)$ observed after graded reductions in myocardial perfusion. The data have been organized into six subgroups similar to the previous illustration. By $5 \mathrm{~min}$ after flow reduction, OC, DP, and $\mathrm{dp} / \mathrm{dt}$ had all declined in proportion to the severity of flow restriction while diastolic pressure was unaffected at this early time period. OC remained stable between 5 and $45 \mathrm{~min}$ as indicated by the fact that mean $\mathrm{OC}$ values in each individual subgroup were within $8 \%$ of each other at all times evaluated $(P>0.05)$. Similarly, DP and dp/dt were also stable for up to $15 \mathrm{~min}$ of reduced perfusion since mean values in individual subgroups did not change by $>10 \%$ over this time period. However, DP and its first derivative increased by $29-42 \%$ from 15 to $45 \mathrm{~min}$ in hearts with the lowest rates of oxidative metabolism $(P<0.05)$. The maximum change occurred at the lowest flow rate with developed pressure and $\mathrm{dp} / \mathrm{dt}$ increasing from $6.2 \pm 1.0 \mathrm{mmHg}$ and $120 \pm 20 \mathrm{mmHg} \mathrm{s}^{-1}$ at $15 \mathrm{~min}$ to $8.8 \pm 1.1$ $\mathrm{mmHg}$ and $155 \pm 26 \mathrm{mmHg} \mathrm{s}^{-1}$ at $45 \mathrm{~min}$, respectively. These data indicate that the response of myocardial $O C$ and systolic ventricular performance to partial, sustained reductions in myocardial perfusion is to establish a new, reduced, steady state rate of oxidative metabolism and contractile function that is stable for up to $\mathbf{4 5} \mathrm{min}$ except for a slight, late increase in systolic function under the most severely flow-restricted conditions.
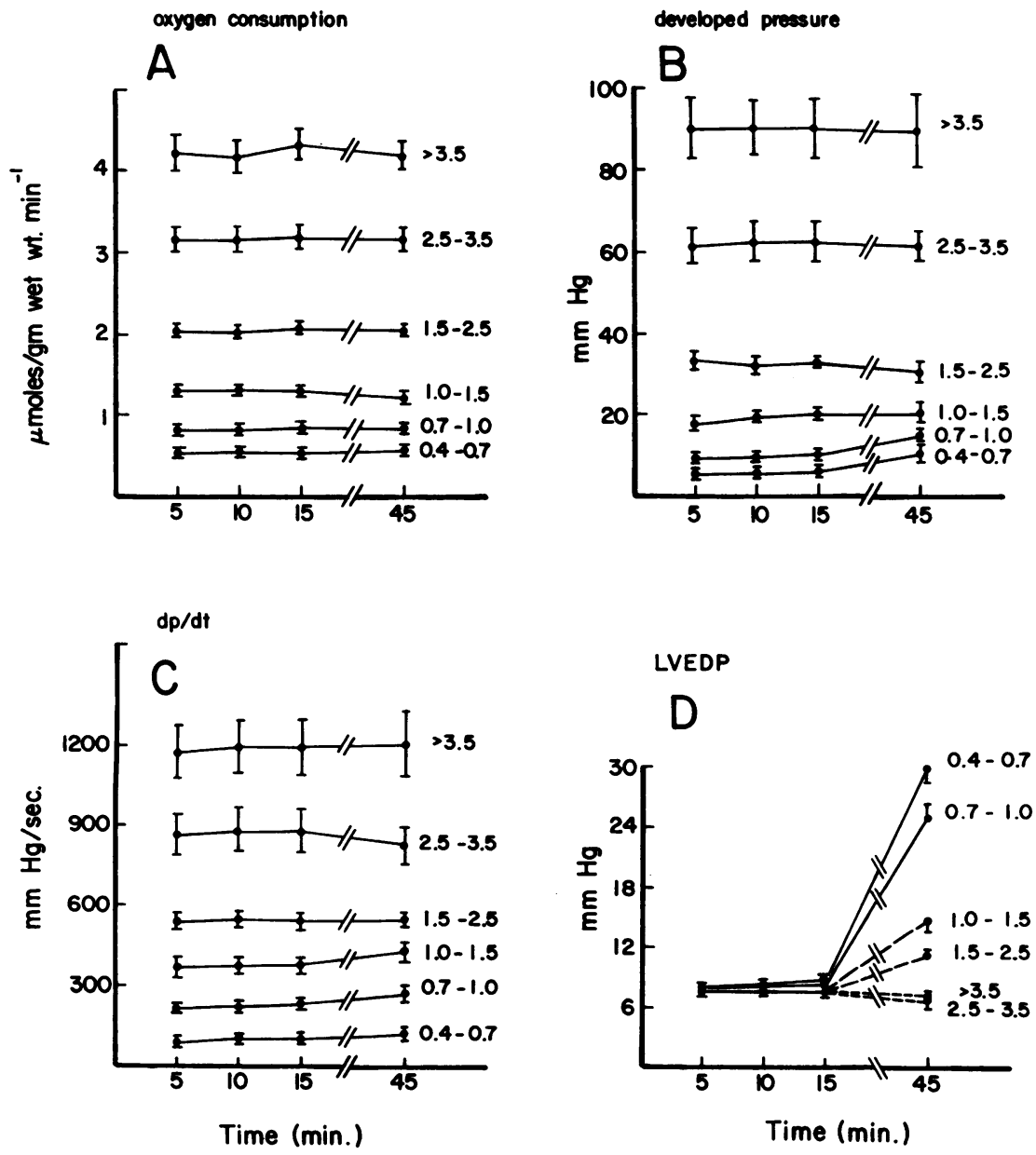

Figure 2. Effect of time on the responses of myocardial OC $(A)$, DP $(B)$, peak dp/dt $(C)$, and enddiastolic pressure $(D)$ to graded, steady-state reductions in myocardial perfusion. Data during the first $15 \mathrm{~min}$ represent the average values for both group I and group II hearts (no significant differences were detected for any of the measured parameters when comparing group I and II hearts during the first $15 \mathrm{~min}[P>0.05$ for all comparisons]). The 45-min values are from single measurements performed in group II hearts. LVEDP, left ventricular end-diastolic pressure. 
Although reducing myocardial perfusion had little effect on diastolic pressure at $5 \mathrm{~min}$, end-diastolic pressure increased by as much as $251 \%$ between 5 and $45 \mathrm{~min}$ in hearts studied during marked reductions in oxidative metabolism $(P<0.05)$. The maximum increase occurred at the lowest flow rate, with diastolic pressure increasing from $8.4 \pm 0.60 \mathrm{~mm} \mathrm{Hg}$ at $5 \mathrm{~min}$ to $13.4 \pm 2.1$ and $29.5 \pm 4.1 \mathrm{mmHg}$ at 15 and $45 \mathrm{~min}$, respectively. Since ventricular loading conditions were kept constant in these experiments, the marked change in diastolic pressure during reduced myocardial perfusion reflects a primary decrease in myocardial compliance that was not accompanied by detectable evidence of deteriorating systolic function.

Fig. 3 illustrates the relationship between isovolumic DP and myocardial $O C$ ( $A$ and $C)$ and $\mathrm{dp} / \mathrm{dt}$ and $O C(B$ and $D)$ at 15 and $45 \mathrm{~min}$ after flow reduction. Each data point represents an individual heart and the solid or dashed straight lines are the least-squares determined best-fit relationships between the systolic functional parameters and myocardial OC. At $15 \mathrm{~min}$, a strong linear correlation exists between both DP and OC $(r$ $=0.94)$ and $\mathrm{dp} / \mathrm{dt}$ and $\mathrm{OC}(r=0.95)$. In addition, although both DP and its first derivative tended to increase slightly at low flows, no significant differences were observed in the linear functions relating DP $(r=0.90)$ and $\mathrm{dp} / \mathrm{dt}(r=0.91)$ to myocardial oxygen at 45 min compared with those obtained at 15 $\min (P>0.05)$. Since the rate $\times$ pressure product and DP changed concordantly with the constant rate stimulation employed in this study, these data indicate that stable, linear relationships exist between isovolumic DP, its first derivative, and the rate $\times$ pressure product versus myocardial $O C$ for up to 45 min after graded flow restrictions.

To evaluate the independent role of reduced myocardial perfusion (i.e., the "garden hose" effect [19]) on the relationships observed between DP, dp/dt, and OC, five hearts were evaluated under constant flow conditions in which myocardial oxygen delivery was progressively reduced by decreasing perfusate hematocrit from 20 to $10 \%, 5 \%$, and $0 \%$ (Fig. 4). As hematocrit was reduced, perfusion rate accelerated slightly from $2.21 \pm 0.14 \mathrm{ml} / \mathrm{g}$ wet wt $\mathrm{min}^{-1}$ to $2.52 \pm 0.10 \mathrm{ml} / \mathrm{g}$ wet $\mathrm{wt}$ $\min ^{-1}$, with no change in relative perfusion pressure (the acceleration of blood flow with reduced hematocrit was presumably due to the diminished viscosity of red blood cell free perfusate, suggesting that altered perfusate viscosity has a minor effect on our pump flow-delivery). No significant differences in the linear relationships between DP and OC $(r=0.94)$ and dp/dt and OC $(r=0.91)$ were observed in this group of hearts compared with that previously observed during progressive flow restriction ( $P>0.05$ for both comparisons), despite the marked differences in coronary flow rate. These data indicate that the linear correlation between isovolumic systolic function and OC is independent of the absolute rate of myocardial perfusion developed pressure

A

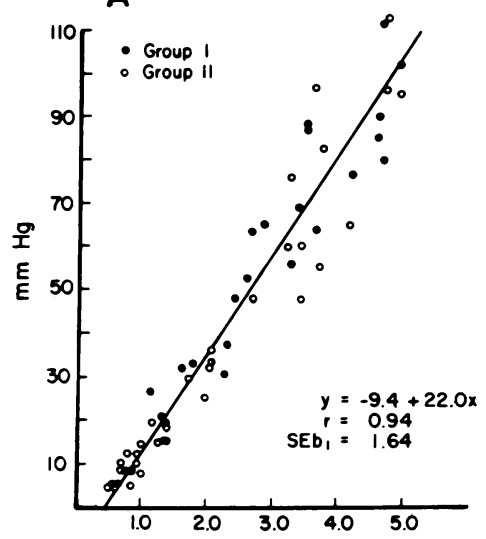

developed pressure

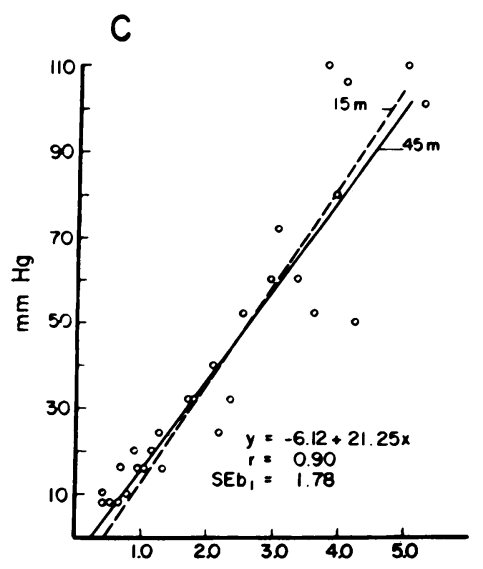

Oxygen Consumption $\mu$ moles/gm wet wt. $\mathrm{min}^{-1}$ $d p / d t$

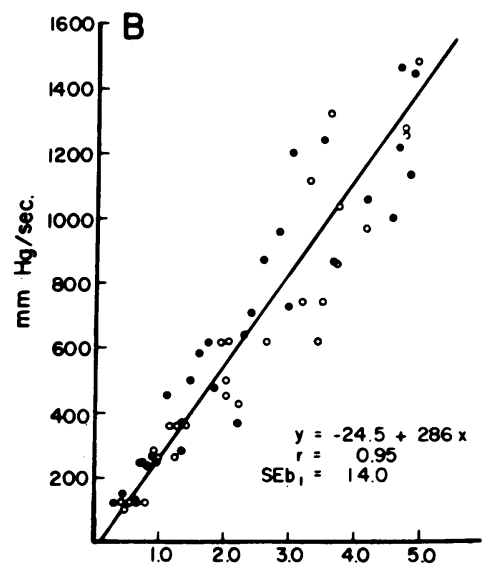

$d p / d t$

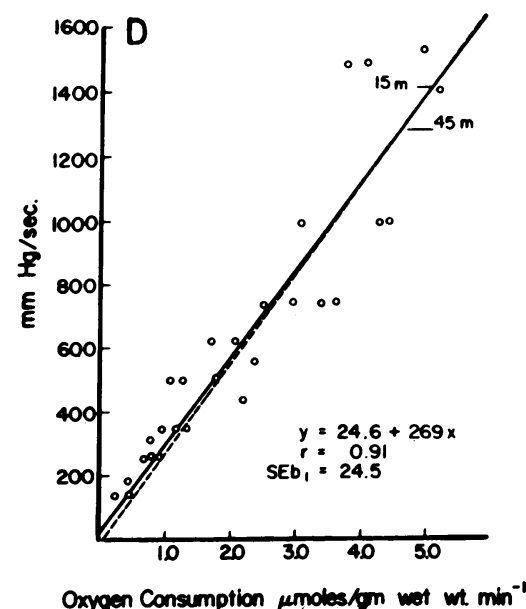

Figure 3. Relationships between DP and peak dp/dt vs. $O C$ at 15 and 45 min after graded flow restriction. The data presented in $A$ and $B$ represent the average of three measurements obtained over the first $15 \mathrm{~min}$ in individual group I (•) and group II (o) hearts. In $C$ and $D$, the data are from single measurements performed at $\mathbf{4 5} \mathrm{min}$ in individual group II hearts. The solid lines are the best-fit straight lines obtained at $15(A$ and $B)$ and $45 \mathrm{~min}(C$ and $D$ ) that were not statistically different $(P$ $>0.05$ ). The linear relationship observed at 15 min has been superimposed on the 45-min data (dashed line, $C$ and $D$ ). 
developed pressure

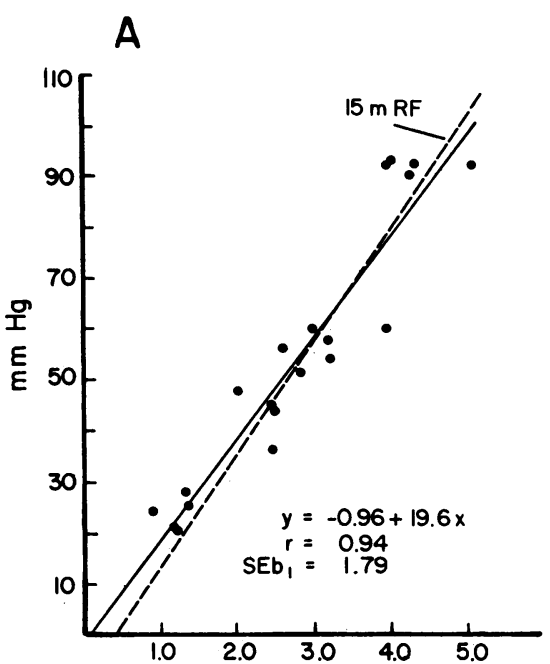

Oxygen Consumption $\mu$ moles/gm wet $\omega t . \mathrm{min}^{-1}$ $d p / d t$

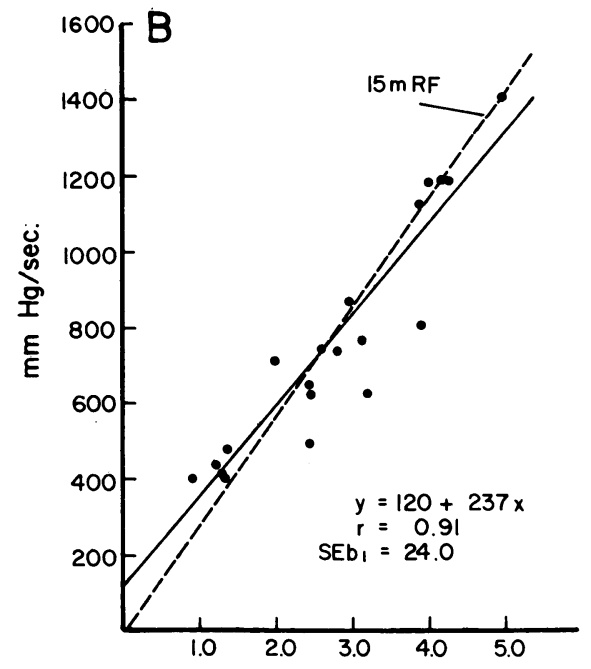

Oxygen Consumption $\mu$ moles/gm wet wt. $\min ^{-1}$
Figure 4. Relationships between DP $(A)$ and peak dp/dt $(B)$ vs. OC in five hearts subjected to progressive restriction of oxygen delivery by staged reductions in perfusate hematocrit while holding flow constant. The data represent single measurements obtained 10-15 min after each hematocrit reduction. The solid lines are the best-fit relationships for the two systolic functional parameters vs. OC. The dashed lines are the best-fit straight lines observed at 15 min during progressive flow restriction (from Fig. 3). No significant differences were observed in the linear relationships between DP, dp/dt, and OC, whether oxygen delivery was restricted by reducing flow or perfusate hematocrit $(P$ $>0.05$ for both comparisons). RF, Reduced flow. and that the garden hose effect does not appear to contribute significantly to this relationship.

To evaluate the potential role of anaerobic metabolism in overall myocardial energy production during progressive flow restriction, myocardial lactate production and oxygen consumption were simultaneously evaluated in 15 hearts during graded, steady state reductions in myocardial perfusion (Table I). As flow and oxidative metabolism were reduced, lactate production and the percent ATP derived from anaerobic metabolism increased. At the lowest rate of oxidative metabolism $\left(0.72 \pm 0.04 \mu \mathrm{mol} / \mathrm{g}\right.$ wet $\left.\mathrm{wt} \mathrm{min}^{-1}\right)$, anaerobic metabolism contributed a maximum of $10.4 \%$ to the total myocardial ATP production. Since anaerobic energy production could account for only $10 \%$ of overall ATP production even at low rates of $\mathrm{OC}$, these data indicate that oxidative energy production remained the predominant energy source during progressive

Table I. Contribution of Anaerobic Metabolism to Total ATP Production

\begin{tabular}{cccr}
\hline OC subgroup & OC & Lactate production & $\begin{array}{c}\text { \% ATP from } \\
\text { lactate }\end{array}$ \\
\hline & $\mu$ mol/g wet wt min $^{-1}$ & $\mu$ mol $/ 8$ wet wt min $^{-1}$ & \\
$1.5-2.5$ & $2.08 \pm 0.06$ & $-0.03 \pm 0.03$ & $<1.0$ \\
$1.0-1.5$ & $1.19 \pm 0.04$ & $-0.32 \pm 0.05$ & 4.5 \\
$0.6-1.0$ & $0.72 \pm 0.04$ & $-0.50 \pm 0.04$ & 10.4
\end{tabular}

Data from 15 hearts (five hearts per group) in which lactate production and $O C$ were assessed simultaneously during partial flow reduction. Measurements were taken under steady-state conditions $15 \mathrm{~min}$ after flow manipulation. Total ATP production was computed from both the $\mathrm{OC}$ values (assuming a $\mathrm{P} / \mathrm{O}$ ratio of 3 ) and from lactate production (assuming $1 \mathrm{~mol}$ of ATP production per mole of lactate produced). These computations also assume negligible steady-state free fatty acid and glycogen metabolism (if significant fatty acid or glycogen metabolism is assumed, the maximum percent of ATP derived from lactate increases to 11.4 and $14.7 \%$, respectively). The - signifies lactate production. Arterial lactate concentration (from red cell glycolysis) averaged $0.25 \pm 0.03 \mathrm{mM}$. Data are mean \pm SEM. flow restriction and that the linear relationship between systolic function and $\mathrm{OC}$ would not be substantially affected by inclusion of anaerobic metabolism.

Fig. 5 illustrates the relationship between myocardial $O C$ and tissue $\operatorname{ATP}(A), \operatorname{CrP}(B)$, total adenine nucleotide (TAN) $(C)$, and lactate $(D)$ content observed 15 min after graded flow restriction (group I). Fig. 6 illustrates similar relationships obtained at $45 \mathrm{~min}$ (group II). The effect of progressive flow restriction on tissue ADP and AMP content has not been illustrated, since these parameters were not altered by reducing the rate of oxidative metabolism. The data presented in Figs. 5 and 6 have also been summarized in Table II.

In contrast to the results obtained for ventricular performance, the relationships between myocardial $O C$ and tissue ATP, CrP, TAN, and lactate content were curvilinear at 15 min with high energy phosphate and lactate content remaining at or near control levels despite substantial reductions in the rate of oxidative metabolism. Comparison of the data obtained at $15 \mathrm{~min}$ (Fig. 5) and $45 \mathrm{~min}$ (Fig. 6) reveals that no significant differences in the curvilinear expressions relating myocardial ATP, CrP, and TAN content to OC were observed at these two time periods ( $P>0.05$ for each comparison). Only tissue lactate demonstrated a consistent change between 15 and $45 \mathrm{~min}$, with a small increase in lactate accumulation detected at the later time period $(P<0.05)$.

Based on the statistical similarity of the expressions relating tissue energy stores to $O C$ at 15 and $45 \mathrm{~min}$, the high energy phosphate data from these two time periods were combined to estimate the rate of restricted oxidative metabolism at which significant high energy phosphate depletion was first observed (Table II). Since lactate content changed with time, the lactate data were not combined and these two time periods were evaluated separately. Analysis of the combined high energy phosphate data revealed that significant ATP, CrP, and TAN depletion were detected when the rate of $O C$ was reduced by $70 \%$ from a control value of $4.25 \pm 0.21 \mu \mathrm{mol} / \mathrm{g}$ wet $\mathrm{wt} \mathrm{min}^{-1}$ to $1.30 \pm 0.04 \mu \mathrm{mol} / \mathrm{g}$ wet $\mathrm{wt} \mathrm{min}^{-1}$. Similarly, significant lactate accumulation was observed at both 15 and $45 \mathrm{~min}$ when the rate of $\mathrm{OC}$ was reduced by $\sim 50 \%$. Taken together, the data depicted in Figs. 5 and 6 and Table II indicate that there is a 

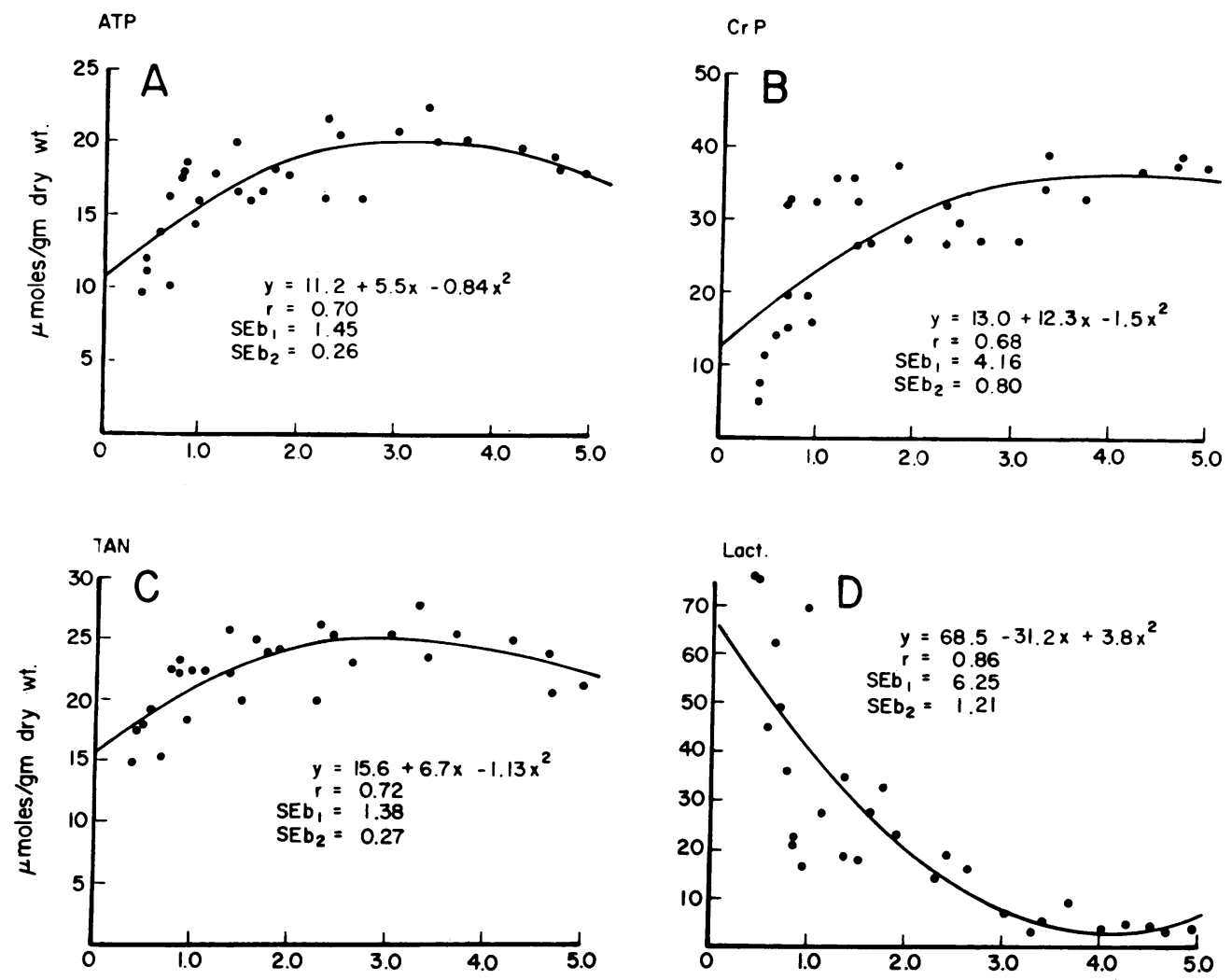

Oxygen Consumption $\mu$ moles/gm wet wt. $\mathrm{min}^{-1}$

Oxygen Consumption rmoles/gm wet wt. $\mathrm{min}^{-1}$

Figure 5. Relationships between tissue ATP $(A), \mathrm{CrP}$ $(B)$, TAN $(C)$, and lactate $(D)$ content vs. myocardial $\mathrm{OC}$ at $15 \mathrm{~min}$ after graded flow restrictions. Data are from individual group I hearts and the solid curves represent the least-squares determined best-fit polynomial expressions.

ATP

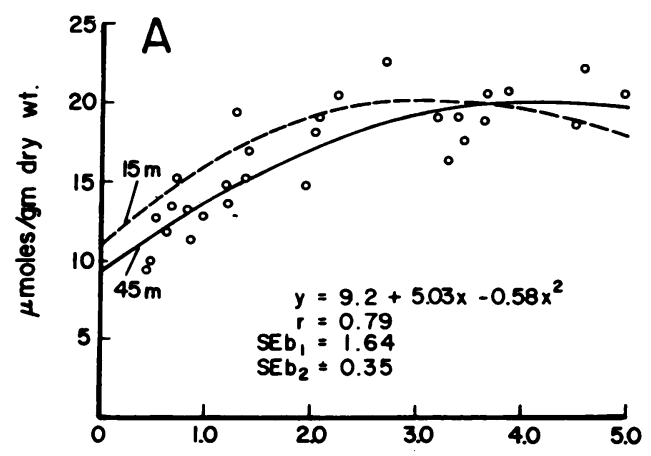

CrP
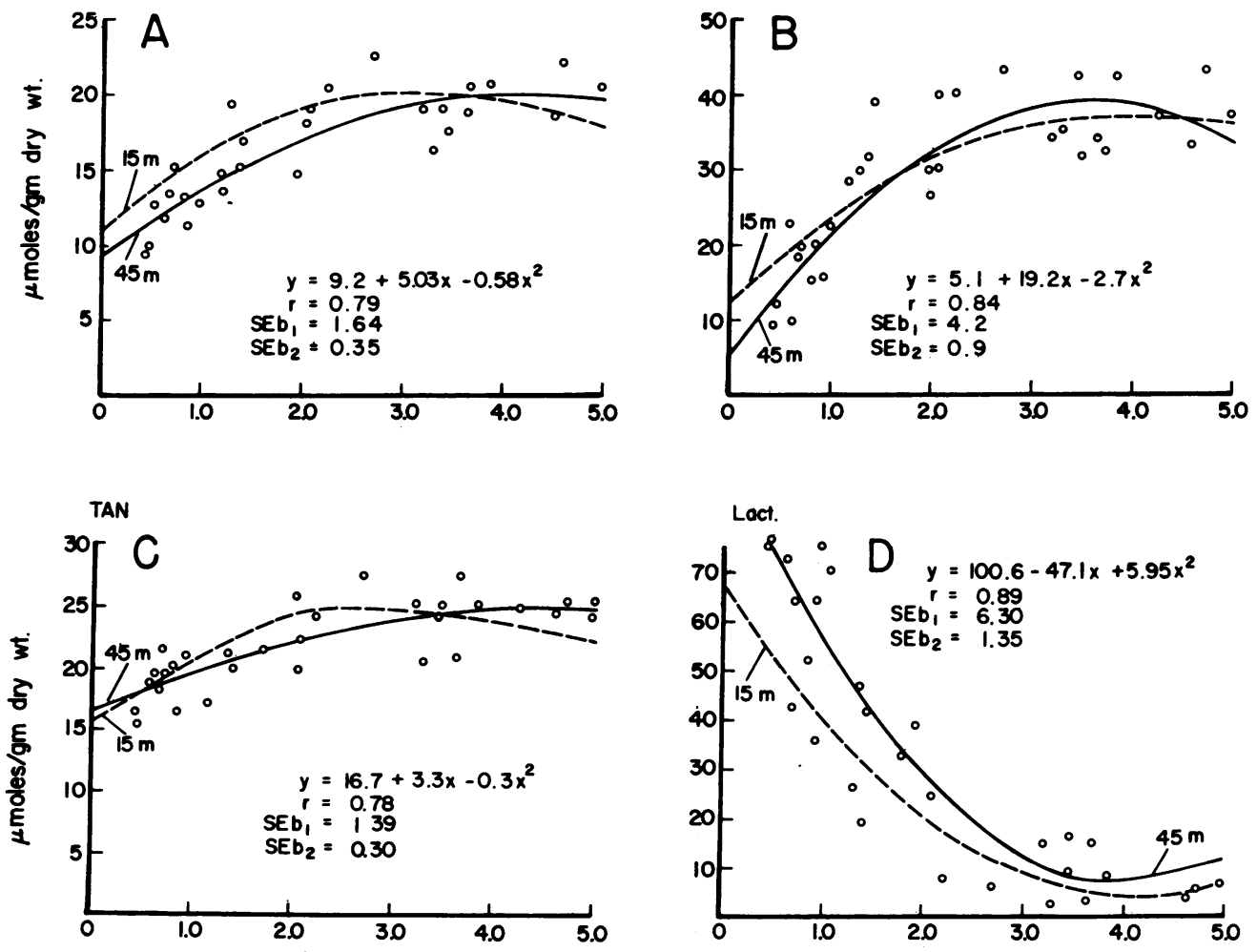

Figure 6. Relationships between tissue ATP $(A), \mathrm{CrP}$ $(B)$, TAN $(C)$, and lactate (D) content vs. myocardial $O C$ at 45 min after graded flow restriction (group II hearts). Each data point is from an individual heart.

The solid curves represent the least-squares determined best-fit polynomial expressions for the 45-min (m) data; the superimposed dashed curves are those observed at 15 min (Fig. 5). The only significant difference observed between 15and 45-min curves was for tissue lactate content $(P$

Oxygen Consumption $\mu$ moles/gm wet wt. $\mathrm{min}^{-1}<0.05$ ). 
Table II. Combined 15- and 45-min Values for OC, DP, and Tissue High Energy Phosphate and Lactate Content

\begin{tabular}{|c|c|c|c|c|c|c|c|}
\hline OC group & OC & DP & ATP & $\mathrm{CrP}$ & TAN & Lactate, $15 \mathrm{~min}$ & Lactate, $45 \mathrm{~min}$ \\
\hline & $\begin{array}{c}\mu \mathrm{mol} / \mathrm{g} \\
\text { wet wt } \mathrm{min}^{-1}\end{array}$ & $m m H g$ & $\mu \mathrm{mol} / \mathrm{g} d r y \mathrm{wt}$ & $\mu m o l / g d r y w t$ & $\mu \mathrm{mol} / \mathrm{g} d r y \mathrm{wt}$ & $\mu m o l / g d r y w t$ & $\mu \mathrm{mol} / \mathrm{g} d r y w t$ \\
\hline$>3.5$ & $4.25 \pm 0.21$ & $90.7 \pm 7.1$ & $19.4 \pm 0.24$ & $38.0 \pm 0.53$ & $24.0 \pm 0.41$ & $5.9 \pm 0.56$ & $7.9 \pm 0.79$ \\
\hline $2.5-3.5$ & $3.17 \pm 0.11$ & $62.0 \pm 4.2$ & $19.5 \pm 0.40$ & $37.3 \pm 0.90$ & $25.1 \pm 0.37$ & $8.1 \pm 1.0$ & $10.2 \pm 1.1$ \\
\hline $1.5-2.5$ & $2.06 \pm 0.07$ & $32.7 \pm 2.0$ & $17.9 \pm 0.35$ & $34.1 \pm 0.85$ & $23.1 \pm 0.29$ & $23.7 \pm 1.3^{*}$ & $31.9 \pm 2.4^{*}$ \\
\hline $1.0-1.5$ & $1.30 \pm 0.04$ & $19.2 \pm 1.4$ & $17.0 \pm 0.32^{*}$ & $31.5 \pm 0.80^{*}$ & $21.9 \pm 0.23^{*}$ & $24.9 \pm 1.2^{*}$ & $34.6 \pm 2.0^{*}$ \\
\hline $0.7-1.0$ & $0.86 \pm 0.03$ & $11.2 \pm 0.9$ & $15.5 \pm 0.21^{*}$ & $22.7 \pm 0.85^{*}$ & $20.9 \pm 0.21^{*}$ & $40.1 \pm 1.8^{*}$ & $56.3 \pm 1.9^{*}$ \\
\hline $0.4-0.7$ & $0.54 \pm 0.03$ & $7.5 \pm 0.5$ & $12.4 \pm 0.29^{*}$ & $14.6 \pm 0.70^{*}$ & $17.8 \pm 0.22^{*}$ & $61.6 \pm 1.2^{*}$ & $67.5 \pm 1.4^{*}$ \\
\hline
\end{tabular}

Combined 15- and 45-min data for OC, DP, and tissue ATP, CrP, TAN, and lactate content. Each data point is the mean \pm SEM for 9 to 12 hearts. * $P<0.05$ vs. control $\left(\mathrm{OC}>3.5 \mu \mathrm{mol} / \mathrm{g}\right.$ wet $\left.\mathrm{wt} \mathrm{min}^{-1}\right)$.

relative preservation of tissue energy stores and lactate content during progressively restricted oxidative metabolism for up to $45 \mathrm{~min}$ after graded flow reductions.

The apparent steady state for tissue high energy phosphate content at normal or slightly reduced levels between 15 and 45 min after partial coronary flow disruption stands in contrast to the progressive depletion of adenine nucleotides previously observed after total coronary artery obstruction. To evaluate the effect of more severe oxygen deprivation on tissue high energy phosphate and lactate content, studies were repeated at 15 and $45 \mathrm{~min}$ after complete cessation of coronary blood flow (Table III). In agreement with previous reports (7-11), myocardial ATP, ADP, and TAN were progressively depleted while AMP increased over this time period, indicating the comparability of the current experimental preparation to those previously employed.

Fig. 7 demonstrates the relationships between isovolumic DP and tissue ATP $(A), \operatorname{CrP}(B)$, TAN $(C)$, and lactate $(D)$ content derived from the combined 15- and 45-min data. Although not shown, similar results were obtained when $\mathrm{dp} / \mathrm{dt}$ was plotted against tissue high energy phosphate and lactate content. In all cases, the relationships between DP and tissue energy metabolites were best described by an exponential expression with substantial reductions in isovolumic pressure development occurring before detectable high energy phosphate depletion or lactate accumulation. Using an analysis similar to that employed for myocardial oxygen consumption vs. tissue energy stores (Table II), DP declined by $80 \%$ from a control value of $90.7 \pm 7.1$ to $19.2 \pm 1.4 \mathrm{mmHg}$ before myocardial ATP, CrP, or TAN content became abnormal. Similarly, pressure development decreased by $64 \%$ to $32.7 \pm 2.0 \mathrm{mmHg}$ before abnormal myocardial lactate content was observed. These data indicate that tissue high energy phosphate stores and lactate content are maintained at or near normal levels in the presence of progressive contractile dysfunction after graded reductions in coronary blood flow.

\section{Discussion}

The results of this study reveal that progressive flow restriction produces parallel reductions of contractile performance and myocardial OC without significant high energy phosphate depletion, except under the most severely flow-limited conditions. These observations suggest that mechanical function is closely coupled to the rate of oxidative energy production during partial coronary flow disruption. Since mechanical energy consumption declined concordantly with DP with the constant rate stimulation imposed here, the preservation of tissue energy stores observed during moderate flow limitation might be a consequence of the apparent coupling of mechanical energy consumption and oxidative energy production, which could function to maintain myocardial energy balance.

During moderate flow restriction, the mechanism whereby mechanical function might be coupled to oxidative energy production does not appear to be depend on detectable abnormalities in myocardial cellular washout, calcium delivery, or whole tissue high energy phosphate content. Specifically, the linear relationship between mechanical performance and $O C$ was observed whether oxygen delivery was restricted by reducing perfusion rate or by reducing perfusate hematocrit without altering blood flow. In addition, the relationship between tissue lactate content and contractile function was definitely nonlinear and qualitatively similar to that observed for high energy phosphates. These observations suggest that flow-related changes in calcium delivery and/or tissue wash-out of metabolic end-products $(20,21)$ do not account for the appar-

Table III. Tissue High Energy Phosphate and Lactate Content during No-Flow Ischemia

\begin{tabular}{|c|c|c|c|c|c|c|}
\hline & ATP & $\mathrm{CrP}$ & ADP & AMP & TAN & Lactate \\
\hline & $\mu \mathrm{mol} / \mathrm{g} d r y \mathrm{wt}$ & $\mu \mathrm{mol} / \mathrm{g} d r y \mathrm{wt}$ & $\mu \mathrm{mol} / \mathrm{g} d r y w t$ & $\mu \mathrm{mol} / \mathrm{g} d r y \mathrm{wt}$ & $\mu \mathrm{mol} / \mathrm{g} d r y \mathrm{wt}$ & $\mu \mathrm{mol} / \mathrm{g} d r y w t$ \\
\hline $15 \mathrm{~min}$ & $8.6 \pm 0.20$ & $<3.0$ & $6.2 \pm 0.21$ & $1.95 \pm 0.08$ & $16.7 \pm 0.32$ & $138.8 \pm 0.90$ \\
\hline $45 \mathrm{~min}$ & $<1.5^{*}$ & $<3.0$ & $3.3 \pm 0.16^{*}$ & $6.3 \pm 0.16^{*}$ & $9.7 \pm 0.20^{*}$ & $162.8 \pm 2.1^{*}$ \\
\hline
\end{tabular}

Tissue high energy phosphate and lactate content at 15 and $45 \mathrm{~min}$ after production of no-flow ischemia. Each data point is the mean \pm SEM for four to five hearts. ${ }^{*} P<0.05$ for 45 - vs. 15 -min values. 

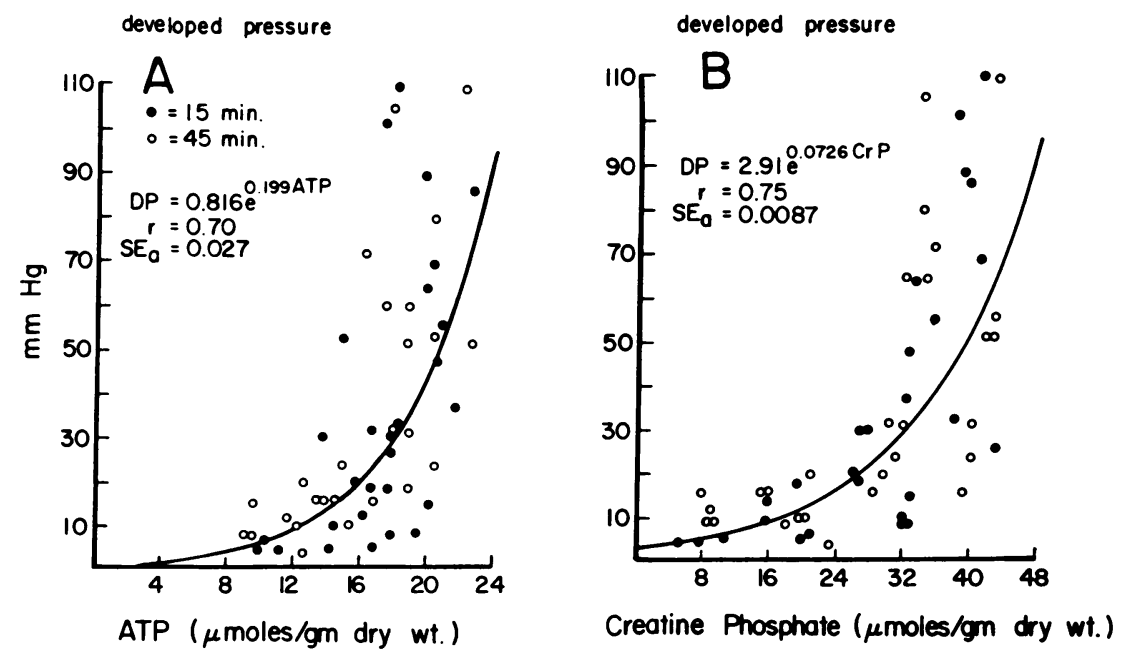

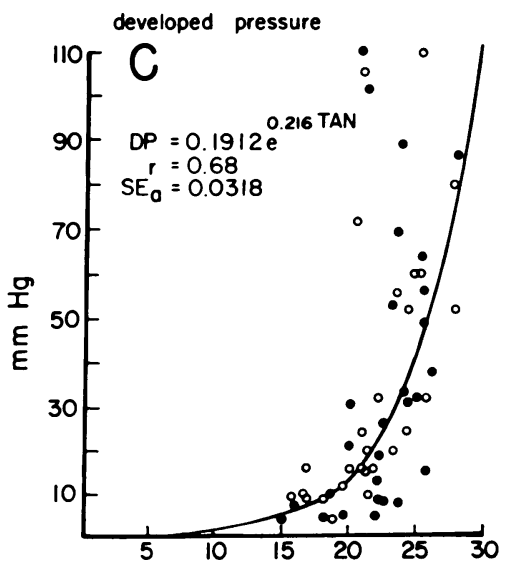

TAN ( $\mu$ moles/gm dry wt.)

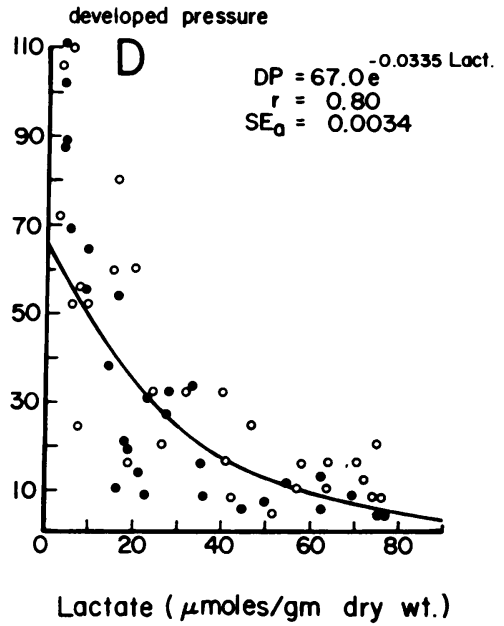

Figure 7. Relationships between DP and tissue $\operatorname{ATP}(A), \operatorname{CrP}(B)$, TAN $(C)$, and lactate $(D)$ content based on the combined 15- and 45-min data. ent coupling of mechanical function to oxidative energy production. The absence of a detectable reduction in whole tissue ATP or CrP content mitigates against depletion of myocardial energy stores directly regulating flow-limited contractile function (11). In addition, the preservation of myocardial high energy phosphate content suggests that modulation of contractile protein function by whole tissue inorganic phosphate accumulation is unlikely (22). Although it is possible that diminished tissue $\mathrm{pO}_{2}$ (13) or altered cyclic AMP content (23) might have affected contractile performance, the precise mechanisms whereby tissue $\mathrm{pO}_{2}$ directly affects contractility and changes in cyclic AMP content during diminished oxidative energy production could explain the parallel reduction in mechanical function have not been published. Furthermore, the strong linear relationship observed between ventricular performance and $O C$ suggests that a decreased supply of oxidatively produced ATP might have reduced contractile function, possibly by depleting ATP in a strategically placed, contractile protein-related microcompartment (24). Compartmentalized ATP depletion could have affected contractile protein function through local $\mathrm{pH}$ changes, phosphate accumulation, or a concentration-dependent reduction in the free energy of ATP hydrolysis (27) without altering whole tissue high energy phosphate or lactate content.
Although tissue energy stores were preserved during moderate flow reduction, more marked restrictions in myocardial perfusion produced high energy phosphate depletion. That the same linear correlation between mechanical activity and $O C$ existed at both moderate and more marked degrees of flow limitation suggests that the development of abnormal tissue high energy phosphate and lactate content might have contributed insignificantly to the depressed function observed at the lower flow rates. In addition, the parallel decline in mechanical function and oxidative metabolism over the entire range of perfusion rates evaluated suggests that the high energy phosphate depletion observed at the lower flow rates does not appear to be secondary dissociation of mechanical energy requirements from oxidative energy production. Other explanations for the high energy phosphate depletion during marked reductions in flow and oxidative metabolism include a relative increase in the percent of fixed, nonmechanical energy consumption, severely impaired tissue wash-out (25), and/or reduction of oxygen delivery below some critical level required to maintain mitochondrial function (26).

The close association of mechanical energy consumption and oxidative energy production with preserved tissue energy stores during partial coronary flow restriction is consistent with a recently reported study from this laboratory evaluating 
myocardial energy balance during excessive inotropic stimulation at basal coronary flow rates (16). In this latter investigation, inotropic stimulation at constant flow rates did not alter the rate $X$ pressure or deplete tissue ATP or CrP content compared with control values. These observations were interpreted to indicate that the myocardium "down-regulates" contractile function in the presence of inadequate coronary flow reserve to preserve myocardial energy balance. In the current study, the apparent coupling of contractile function to oxidative energy production suggests that the previously observed downregulation of mechanical function during positive inotropic stimulation might have been due to the constant myocardial oxygen supply and limited ability to increase oxidative energy production. Taken together, these two studies suggest that the close association of contractile performance and OC might be observed under several different conditions of limited oxygen supply and/or altered oxygen demand and might indicate a limited role for excessive mechanical energy consumption in the depletion of myocardial high energy phosphate stores during incomplete coronary flow disruption.

Comparison of the current results to previous studies evaluating the relationship between ischemic contractile dysfunction and directly measured tissue high energy phosphate stores is limited by substantial differences in the experimental models employed. In many of these prior investigations, severe oxygen deprivation was produced by acutely occluding a coronary artery or by perfusing isolated hearts with oxygen-free perfusate (7-11). Under these conditions, myocardial OC becomes rapidly and severely depressed and technically unquantifiable, making it difficult to evaluate the role of a diminished rate of oxidative ATP synthesis in the production of hypoxic contractile failure. In addition, myocardial contraction rapidly ceases and tissue high energy phosphates are progressively depleted without the reestablishment of either a functional or metabolic steady state. In contrast, in the current model of partial coronary flow disruption, $\mathrm{OC}$ could be quantified and the maintenance of a reduced rate of oxidative energy production appeared to produce a reasonably stable preparation in which time-dependent changes in myocardial function and energy metabolism were minimal relative to the effects of flow impairment. The presence of continued oxidative metabolism presumably also contributed to the preservation of tissue high energy phosphate content not observed in previous reports evaluating more severe oxygen deprivation.

In a recently published study, the relationships between hypoxic contractile failure, tissue high energy phosphate content, and flux through the creatine kinase reaction were studied in in vivo rat hearts using $\left[{ }^{31} \mathrm{P}\right] \mathrm{NMR}$ techniques (12). In contrast to other previous investigations, the degree of myocardial oxygen deprivation produced in this latter study would appear to be more comparable with that evaluated here. The results obtained during in vivo hypoxic myocardial perfusion revealed that contractile dysfunction was more closely related to flux through the creatine kinase reaction than to whole tissue ATP or CrP content. This observation is consistent with the current results, especially if the proposed coupling of the creatine kinase reaction to oxidative ATP production, mitrochondrial translocation, and mechanical consumption is equally applicable to hypoxic and normoxic conditions. However, unlike the preservation of tissue high energy phosphate content observed here, myocardial ATP and CrP content did not remain at normal levels during in vivo hypoxic myocardial perfusion. The high energy phosphate depletion observed in vivo might reflect the complexity of producing cardiac failure in intact animals due to the combined effect of several compensatory processes including excessive catecholamine stimulation and cardiac dilation in the presence of significant hypotension and altered cardiac perfusion.

The present observation of a linear relationship between contractile performance and oxygen consumption is in qualitative agreement with previously published studies demonstrating a close correlation between the degree of blood flow impairment and the severity of contractile dysfunction during partial coronary artery obstruction (1-4). However, the relative preservation of tissue high energy phosphate stores and limited lactate accumulation does not agree with the results of several previous studies demonstrating a strong correlation between the rate of coronary venous lactate and inosine production and the degree of flow and contractile impairment $(1,5)$. Potential explanations for this apparent discrepancy are that: (a) These previous studies were performed during regional flow reductions in which the relationship between venous metabolite concentration and severity of blood flow reduction is difficult to determine; (b) An increase in the rate of venous lactate and inosine production could reflect regionally improved tissue washout and/or accelerated synthesis and membrane transport with minimal alterations in tissue content; and (c) The amount of inosine detected in venous effluent accounted for a very small portion of myocardial ATP content (5) and increased inosine production could reflect turnover of a small pool of ATP undetectable by whole tissue assays. Therefore, these apparently disparate results could reflect the different methodologies involved to assess myocardial energy metabolism.

Diastolic pressure was observed to increase between 15 and 45 min after flow reduction, with the more marked changes occurring under more severely flow deprived conditions. These increases in diastolic pressure reflect a primary reduction in myocardial compliance under the constant loading conditions imposed in this study. Since corresponding reductions in oxidative ATP production, tissue high energy phosphate content, or systolic function were not observed over this same time period, the reduction in myocardial compliance presumably reflects cellular calcium accumulation due to abnormal calcium homeostasis.

The relative preservation of tissue high energy phosphate content suggests that moderate ventricular hypofunction occurring in vivo after acute, partial coronary artery obstruction might not be associated with ischemic ATP and $\mathrm{CrP}$ depletion or lactate accumulation. In addition, altered ventricular function related to acute interventions designed to improve blood flow or reduce myocardial energy requirements might not reflect quantitatively similar changes in tissue high energy phosphate or lactate content. Therefore, the current results suggest that caution should be exercised in interpreting changes in ventricular function during partial coronary artery obstruction as indicative of corresponding alterations in cellular energy metabolism.

\section{Acknowledgments}

The thoughtful review of this manuscript by Carl Apstein, M.D. is gratefully appreciated. The expert secretarial assistance of Ms. Clau- 
dette Lamothe and illustration preparation by Mr. H. C. Morgan are also acknowledged.

This work was supported by grant HL-30699 from the National Heart, Lung and Blood Institute.

\section{References}

1. Stowe, D. F., D. G. Mathey, W. Y. Moores, S. A. Glantz, R. M. Townsend, P. Kabra, K. Chatterjee, W. W. Parmley, and J. V. Tyberg. 1978. Segment stroke work and metabolism depend on coronary blood flow in the pig. Am. J. Physiol. 234:H597-H607.

2. Gallagher, K. P., M. Matsuzaki, G. Osakada, W. S. Kemper, and J. Ross, Jr. 1983. Effect of exercise on the relationship between myocardial blood flow and systolic wall thickening in dogs with acute coronary stenosis. Circ. Res. 52:715-729.

3. Vatner, S. F. 1980. Correlation between acute reductions in myocardial blood flow and function in conscious dogs. Circ. Res. 47:201-207.

4. Waters, D. D., P. Daley, H. L. Wyatt, H. J. C. Swan, and J. S. Forrester. 1977. Early changes in regional and global left ventricular performance induced by graded reductions in regional coronary perfusion. Am. J. Cardiol. 39:537-543.

5. Willem deJong, J., and S. Goldstein. 1974. Changes in coronary venous inosine concentration and myocardial wall thickening during regional ischemia in the pig. Circ. Res. 35:111-166.

6. Feinstein, M. B. 1962. Effects of experimental congestive heart failure, ouabain, and asphyxia on the high energy phosphate and creatine content of the guinea pig heart. Circ. Res. 10:333-346.

7. Pool, P. E., J. W. Covell, C. A. Chidsey, and E. Braunwald. 1966. Myocardial high energy phosphate stores in acutely induced hypoxic heart failure. Circ. Res. 19:221-229.

8. Jones, C. E., J. X. Thomas, J. C. Parker, and R. E. Parker. 1976. Acute changes in high energy phosphates, nucleotide derivatives and contractile force in ischemic and nonischemic canine myocardium following coronary occlusion. Cardiovasc. Res. 10:275-282.

9. Gudbjarnason, S., P. Mathes, and K. G. Ravens. 1970. Functional compartmentation of ATP and creatine phosphate in heart muscle. J. Mol. Cell. Cardiol. 1:325-339.

10. Dhalla, N. S., J. C. Yates, D. A. Walz, V. A. McDonald, and R. E. Olson. 1972. Correlation between changes in endogenous energy stores and myocardial function due to hypoxia in isolated perfused rat heart. Can. J. Physiol. Pharmacol. 50:333-345.

11. Hearse, D. J. 1978. Oxygen deprivation and early myocardial contractile failure: a reassessment of the possible role of adenosine triphosphate. Am. J. Cardiol. 44:1115-1121.

12. Bittl, J. A., J. A. Balschi, and J. S. Ingwall. 1987. Contractile failure and high energy phosphate turnover during hypoxia: ${ }^{31} \mathrm{P}-\mathrm{NMR}$ surface coil studies in living rat. Circ. Res. 60:871-878.
13. Mathews, P. M., D. J. Taylor, and G. K. Radda. 1986. Biochemical mechanisms of acute contractile failure in the hypoxic rat heart. Cardiovasc. Res. 20:13-19.

14. Bergman, S. R., R. E. Clark, and B. E. Sobel. 1979. An improved isolated heart preparation for external assessment of myocardial metabolism. Am. J. Physiol. 236(Heart Circ. Physiol. 5):H644H651.

15. Apstein, C. S., R. C. Dennis, L. Briggs, W. M. Vogel, J. Frazer, and C. R. Valeri. 1985. Effect of erythrocyte storage and oxyhemoglobin affinity changes on cardiac function. Am. J. Physiol. 248(Heart Circ. Physiol. 17):H508-H515.

16. Marshall, R. C., W. W. Nash, M. M. Bersohn, and G. Wong. 1987. Myocardial energy production and consumption remain balanced during positive inotropic stimulation when coronary flow is restricted to basal rates in rabbit heart. J. Clin. Invest. 80:1165-1171.

17. Falsetti, H. J., R. E. Mathes, R. J. Carroll, R. L. Gupta, and A. C. Bell. 1974. Analysis and correction of fluid wave distortion in fluid filled catheter systems. Circulation. 49:165-172.

18. Bergmeyer, H. 1974. Methods in Enzymatic Analysis. Academic Press, Inc., New York.

19. Vogel, W. M., C. S. Apstein, L. L. Griggs, W. H. Gaasch, and B. J. Ahn. 1982. Acute alterations in left ventricular diastolic chamber stiffness: role of the "erectile" effect or coronary arterial pressure and flow in normal and damaged hearts. Circ. Res. 51:465-478.

20. Katz, A. M. 1968. Effects of interrupted coronary flow upon myocardial metabolism and contractility. Prog. Cardiovasc. Dis. 10:450-465.

21. Katz, A. M., and H. H. Hect. 1969. The early pump failure of the ischemic heart. Am. J. Med. 47:497-502.

22. Kusuoka, H., M. L. Weisfeldt, J. L. Zweier, W. E. Jacobus, and E. Marban. 1986. Mechanism of early contractile failure during hypoxia in rabbit heart: evidence for modulation of maximal $\mathrm{Ca}^{2+}$-activated force by inorganic phosphate. Circ. Res. 59:270-282.

23. Winegrad, S. 1984. Regulation of cardiac contractile proteins: correlations between physiology and biochemistry. Circ. Res. 55:565574.

24. Jacobus, W. E., and J. S. Ingwall, editors. 1980. Heart Creatine Kinase: The Integration of Isozymes for Energy Distribution. Williams \& Wilkins, Baltimore, MD. 1-8.

25. Neely, J. R., and L. W. Grotyohann. 1984. Role of glycolytic products in damage to ischemic myocardium: dissociation of adenosine triphosphate levels and recovery of ventricular function of reperfused hearts. Circ. Res. 55:816-824.

26. Lubbens, D. W., and M. Kessler. 1969. Oxygen supply and rate of tissue respiration. In Oxygen Transport in Blood and Tissue. D. W. Lubbens, U. C. Luft, G. T. Thews, and E. Witzels, editors. Georg Thieme Verlag, Stuttgart, FRG. 90-99.

27. Wilkie, D. R. 1960. Thermodynamics and the interpretation of biological heat measurements. Prog. Biophys. Biophys. Chem. 10:260-298. 